Mots. Les langages du politique

\title{
Plus précoces et moins sanctionnés ? Usages des statistiques dans les discours sur les jeunes face aux drogues
}

Ivana Obradovic et François Beck

\section{CpenEdition}

Journals

Édition électronique

URL : https://journals.openedition.org/mots/20986

DOI : $10.4000 /$ mots. 20986

ISSN : 1960-6001

Éditeur

ENS Éditions

\section{Édition imprimée}

Date de publication : 15 décembre 2012

Pagination : 137-152

ISBN : 978-2-84788-387-9

ISSN : 0243-6450

\section{Référence électronique}

Ivana Obradovic et François Beck, «Plus précoces et moins sanctionnés ? Usages des statistiques dans les discours sur les jeunes face aux drogues », Mots. Les langages du politique [En ligne], 100 | 2012, mis en ligne le 15 décembre 2014, consulté le 23 avril 2022. URL : http:// journals.openedition.org/mots/20986; DOI : https://doi.org/10.4000/mots.20986 


\section{Plus précoces et moins sanctionnés? Usages des statistiques dans les discours sur les jeunes face aux drogues}

La difficulté à rendre compte de l'évolution des usages de stupéfiants tient en partie à leur caractère illicite, qui contribue à les soustraire aux tentatives d'objectivation, mais aussi à la complexité de l'évaluation de l'efficacité des politiques publiques conduites pour faire baisser le nombre de consommateurs, en particulier parmi les jeunes ${ }^{1}$, objectif revendiqué par tous les plans d'action gouvernementaux de lutte contre les drogues élaborés en France depuis 1998 (MILDT, 1999, 2004, 2008). Peu de travaux se sont risqués à proposer une évaluation scientifique des réponses publiques apportées au phénomène de la consommation de drogues, si ce n'est d'un point de vue économique, et leurs résultats sont souvent limités à la mise en œuvre des politiques plus qu'à leur impact observable (Layne et al., 2001 ; Reuter, Greenfield, 2001 ; Setbon et al., 2003; Wood et al., 2003; Kopp, Fenoglio, 2004 ; Degenhardt et al., 2005). Les différents acteurs politiques avancent néanmoins régulièrement des interprétations des tendances observées, imputant volontiers la baisse de tel niveau d'usage à l'impact d'une loi ou au succès d'une campagne d'information récemment menée ${ }^{2}$. À travers les discours politiques sur «la » ou «les » drogue(s)3, il s'agit en effet de nourrir des stratégies de légitimation ou de disqualification des politiques publiques. Compte tenu des difficultés de mesure des usages de drogues, a fortiori en tendance longue, les revendications chiffrées présentes

1. La question du «classement » de la jeunesse soulève des difficultés bien connues sur lesquelles nous ne reviendrons pas dans cet article (Bourdieu, 1984; Chamboredon, 1966; Thévenot, 1979).

2. Voir, par exemple, l'interprétation des chiffres issus de l'enquête ESCAPAD 2011 (menée par l'OFDT auprès des jeunes de 17 ans) par le président de la Mission interministérielle de lutte contre les drogues et les toxicomanies (MILDT). S'inquiétant de la hausse conjuguée de l'usage régulier d'alcool et des ivresses répétées, celui-ci soulignait néanmoins que l'expérimentation de l'alcool et du tabac chez les jeunes avait régressé, en attribuant cette évolution à l'impact des mesures gouvernementales restreignant l'accessibilité de ces produits (France Soir, 8 décembre 2011).

3. La définition du terme drogue ne fait pas consensus (Becker, 2001). Ces questions ont notamment ressurgi lorsqu'il s'est agi de quantifier le phénomène en France, dans les années quatrevingt-dix (Beck, 2005).

Université de Picardie Jules Verne, OFDT, CURAPP

ivobr@ofdt.fr

Cermes3 (Université Paris Descartes, Sorbonne Paris Cité / CNRS UMR 8211 / Inserm U988 / EHESS)

francois.beck@inpes.sante.fr

Mots. Les langages du politique $\mathrm{n}^{\circ} 100$ novembre $2012 \bullet 137$ 
dans les discours politiques renseignent au moins autant sur le phénomène lui-même que sur les stratégies de ceux qui entendent évaluer l'efficacité des politiques publiques.

À travers deux études de cas, cet article prétend contribuer à la réflexion sur l'usage des statistiques dans les discours politiques et la construction de leur(s) signification(s) par le langage. À l'image des discours publics s'alarmant, dès la fin des années soixante, de l'émergence d'usages de drogues au sein de la jeunesse, l'idée d'un supposé « rajeunissement des consommations» semble couramment admise depuis quelques années, arguant notamment que l'expérimentation de cannabis chez les mineurs a doublé en dixans. Cette affirmation mérite cependant d'être nuancée, à la fois en fonction des produits mais aussi au regard de l'évolution plus générale des pratiques des adolescents et des jeunes adultes. Un second exemple reflétant les représentations des problèmes liés aux drogues réside dans les commentaires politiques récurrents jugeant la pénalisation de l'usage de stupéfiants peu effective. Cette vision est alimentée par les partisans d'une application plus rigoureuse de la loi, qui dénoncent une «dépénalisation de fait » et jugent la loi du 31 décembre 1970 interdisant l'usage de drogues mal appliquée, voire permissive4, selon les termes employés par Nicolas Sarkozy, alors ministre de l'Intérieur, en 2003. L'analyse des statistiques policières et judiciaires montre pourtant que, selon la définition retenue de la «pénalisation » de l'usage de stupéfiants, les évolutions observées divergent sensiblement. Qu'elle concerne les niveaux de consommation des jeunes ou l'évolution de la répression pénale à l'égard des consommateurs, l'interprétation politique des évolutions révèle des usages symboliques du chiffre, dès lors que l'analyse pourrait conduire à des conclusions différentes.

L'usage de chiffres dans les discours sur les jeunes face aux drogues s'inscrit par ailleurs dans un contexte où les enjeux de qualification sémantique du «problème de la toxicomanie » déterminent la manière dont les acteurs politiques s'emparent de cette question (Bergeron, 1999)5. À l'image des termes dramatisants employés à la fin des années soixante, contrastant avec la « réalité » perceptible au travers des rares chiffres de consommation disponibles 6

4. Le ministre de l'Intérieur a ainsi évoqué, devant la commission d'enquête sénatoriale de lutte contre les drogues illicites en 2003, "l'existence d'une loi dure, très dure, vidée de son sens par l'application molle, très molle qui en a été faite. La loi est dure, mais elle n'est pas appliquée » (Olin, Plasait, 2003, p. 543). Nous reviendrons sur ce point dans le cours de l'article.

5. Les enjeux de qualification linguistique du «problème de la toxicomanie » ont gouverné, dès l'origine, le processus de construction politique de ce secteur d'action publique. Comme le souligne Bergeron, « le vocabulaire, les arguments et les images mobilisés dans les médias et par le gouvernement de l'époque dépeignent une situation dramatique », illustré par le recours à des métaphores fortement connotées : «fléau national», "catastrophe sans précédent», « risque généralisé», « une jeunesse menacée» (Bergeron, 1999, p. 24). En 1969, le nombre d' «affaires de drogues » est inférieur à 500, pour un total de 1200 personnes interpellées (Lahaye, 1989).

6. Comme le souligne Bergeron (1999, p. 24), «le vocabulaire, les arguments et les images mobilisés dans les médias et par le gouvernement de l'époque dépeignent une situation dramatique ", par le recours à des métaphores fortement connotées : «fléau national», «catastrophe sans 
(Bernat de Célis, 1992), le cadrage du problème de la consommation de drogues par les acteurs politiques convoque souvent, en affinité avec les formes de valorisation médiatique7, un style linguistique métaphorique, mettant en avant l'image de la catastrophe, naturelle (« explosion», «inondation », « déferlante », «tsunami ») ou sanitaire (" épidémie», « peste», « cancer»), suggérant le risque, la menace et l'urgence à agir. Le rapport de la commission d'enquête sénatoriale, paru en 2003, qui constitue une partie du matériau d'analyse de cet article, s'intitulait ainsi : «Drogue : l'autre cancer» (Olin, Plasait, 2003).

L'objectif de cet article n'est pas tant d'approfondir l'analyse des actes du discours ou des registres d'argumentation politique développés autour des chiffres disponibles que de mettre au jour, à partir d'études de cas, les séquences de raisonnement développées autour des données quantitatives publiées. Dans la lignée des travaux d'Alain Desrosières (2004 et 2008), il s'agit de montrer comment certains chiffres sont «factualisés », au sens de Gusfield 8 , pour donner, tour à tour, un caractère général, une valeur d'exemple ou un supplément d'autorité à des arguments relevant d'évaluations biaisées et de jugements politiquement situés. Cette contribution propose donc une réflexion sur les effets d'imposition des statistiques telles qu'utilisées dans les discours politiques, notre approche du discours s'inscrivant dans le domaine de la construction des problèmes publics et portant, plus spécifiquement, sur la dimension symbolique de l'action publique. En commentant quelques exemples où un phénomène est réduit à un seul chiffre, nous verrons comment, au sein de ces séquences de raisonnement, le recours aux chiffres contribue à la fois à produire et à structurer des cadres d'analyse du "problème de la consommation de drogues». Nous essaierons ainsi d'établir comment, dans les discours publics sur la drogue, les chiffres sont instrumentalisés selon un registre confondant souvent énoncés à valeur d'arguments et énoncés purement constatifs, en faisant de la revendication de certains chiffres, plutôt que d'autres, un acte politique à part entière.

précédent», «risque généralisé», «une jeunesse menacée». En 1969, le nombre d'«affaires de drogues » est inférieur à 500, pour un total de 1200 personnes interpellées (Lahaye, 1989).

7. Il suffit, pour s'en convaincre, de citer quelques-unes des «couvertures choc » récurrentes dans la presse depuis trente ans : «Alerte à la drogue» racontant «la mort par overdose de Martine, 17 ans, à Bandol, pendant l'été 69» (Nouvel Observateur, 8 septembre 1969), "Une catastrophe nationale... On meurt de la drogue à 17 ans... » (Le Parisien libéré, 7 avril 1973), «Alerte à la cocaïne chez les jeunes» (Choc, juillet 2007; Phosphore, juin 2009), " “Nous sommes face à un tsunami”. L'analyse d'Étienne Apaire, magistrat et actuel patron de la MILDT » (L'Express, 13 mars 2008).

8. En étudiant le registre d'argumentation et l'arsenal rhétorique mobilisés pour aborder la question de l'alcool au volant aux États-Unis dans les années soixante, Gusfield montre comment « les faits liés aux actions de boire et de conduire sont convertis en problème public de l'alcool au volant» (Gusfield, 1981, trad. fr. 2009, p. 58). La «factualisation » des dangers liés à la conduite sous l'influence de l'alcool consiste à transformer certains éléments de preuve choisis et mis en scène dans des termes dramatisants en un sens commun, reconnu par tous : «de buveur en société, il devient buveur à problèmes» (p.108), voire «ivrogne-tueur» en puissance (p.126). 


\section{Choix des études de cas}

Les exemples analysés ici sont issus de réflexions parlementaires orchestrées à des époques différentes. Nous analyserons d'abord le cadrage sémantique du problème de l'usage de drogues mis en place lors des travaux préparatoires à l'adoption de la loi du 31 décembre 1970. Nous poursuivrons en examinant les termes et les chiffres employés pour qualifier les enjeux publics liés aux drogues au cours de deux épisodes de médiatisation de cette question intervenus au cours de la dernière décennie, à l'occasion de la mise en place de commissions d'enquête parlementaire' 9 visant à étudier la politique gouvernementale de lutte contre les drogues illicites. La première séquence a eu lieu en 2002-2003, lors de la mise en place, par le Sénat, d'une commission d'enquête, composée de 21 membres, sur la politique nationale de lutte contre les drogues illicites, présidée par Nelly Olin, sénatrice UMP du Val-d’Oise. Celle-ci a été chargée d' "établir un diagnostic de la dangerosité des différentes drogues» et d'examiner, plus particulièrement, l'action des administrations et des organismes publics ou associatifs chargés de la mise en œuvre des politiques de répression, de prévention et d'information en matière de toxicomanie, sous l'angle, notamment, de «l'efficacité de l'arsenal pénal réprimant l'usage et le trafic des stupéfiants et son éventuelle adaptation $»^{10}$. La seconde s'est déroulée à partir d'octobre 2010, date de la création d'une mission commune d'information sur les toxicomanies (Sénat-Assemblée nationale), coprésidée par Serge Blisko, député socialiste radical citoyen (SRC) de la $10^{\mathrm{e}}$ circonscription de Paris, et François Pillet, sénateur UMP du Cher. Quinze sénateurs et quinze députés ont alors été désignés pour faire des «propositions réalistes et efficaces pour lutter au mieux contre ce phénomène et apporter aux victimes des réponses appropriées ${ }^{11}$. Chacune de ces instances parlementaires a donné lieu à un rapport public, accessible sur les sites parlementaires ${ }^{12}$, qui a été largement débattu dans la presse (Olin, Plasait, 2003; Branget, Barbier, 2011). Les conclusions de ces rapports parlementaires ont, à chaque fois, été accompagnées d'une liste des personnalités auditionnées et d'un compte rendu de

9. La création d'une commission d'enquête parlementaire, le plus souvent suscitée par les répercussions d'une question dans l'opinion publique, doit permettre de recueillir des éléments d'information, soit sur des faits déterminés, soit sur la gestion des services publics ou des entreprises nationales. Depuis la loi constitutionnelle du 23 juillet 2008 , le rôle des commissions d'enquête a été «constitutionnalisé» afin de faire du contrôle parlementaire un instrument d’évaluation pouvant déboucher sur un travail législatif (Houillon, 2010).

10. Proposition de résolution adoptée au Sénat le 10 juillet 2002, tendant à la création d'une commission d'enquête sur la politique nationale de lutte contre les drogues illicites (présentée par Bernard Plasait et Henri de Raincourt, au nom du groupe des républicains et indépendants).

11. Extrait du communiqué de presse du Sénat daté du 5 octobre 2010.

12. www.senat.fr/commission/enquete/; www.senat.fr/commission/missions/; sites consultés le 20 septembre 2012 . 
ces auditions. Les deux commissions d'enquête ont également en commun d'avoir ouvert un débat public sur la réforme de la loi de 1970, même s'il s'est conclu par la décision de ne pas modifier le cadre légal.

\section{De la rhétorique du «fléau social " à la preuve par le chiffre : vicissitudes des argumentations politiques autour des usages de drogues}

La quantification ${ }^{13}$ des usages de drogues ne semblait pas constituer une priorité à la fin des années soixante : l'objectif avoué des pouvoirs publics était alors l'éradication des usages de drogues ${ }^{14}$. Quelle qu'en fut l'ampleur réelle, la crainte de la contagion tenait alors lieu d'argument en soi, justifiant une intervention publique volontariste. La nécessité de disposer d'indicateurs de mesure de l'ampleur du phénomène et de son évolution était pourtant reconnue, comme en témoignent les propos sur le "problème de la drogue »15 tenus en octobre 1969 par Pierre Mazeaud, membre de l'Union des démocrates pour la République :

La découverte de plusieurs affaires de drogues a brusquement fait apparaître à l'opinion publique l'ampleur du problème. En réalité, depuis plusieurs années, on a pu observer l'inquiétante progression de la consommation de stupéfiants chez les jeunes. (Compte rendu intégral de la séance de l'Assemblée nationale du 24 octobre 1969, Journal officiel du 25 octobre 1969, p. 2931)

Le député fait allusion aux décès par overdose survenus au cours de l'été 1969, à la suite du «drame de Bandol ${ }^{16}$. En établissant un lien quasi mécanique entre faits divers ("plusieurs affaires de drogues») et mesure du phénomène («l'ampleur du problème»), Pierre Mazeaud résume l'amalgame fréquemment opéré dans le discours politique entre des situations particulières et des tendances observables en population générale. Cette affirmation, présentée comme un constat irréfutable, inspire un diagnostic général sur l'accélération présumée des usages de drogues au sein d'une catégorie de population spécifique, les jeunes.

13. Nous employons ici le terme de quantification au sens où l'entend Alain Desrosières, à savoir la transformation, par une procédure conventionnelle, d'un matériel exprimé par le langage en valeurs quantitatives (Desrosières, 2008).

14. Un exemple de cette représentation du problème peut être trouvé dans les termes de la circulaire du ministère de la Santé Publique relative au rôle de la santé scolaire dans la lutte contre la toxicomanie datée du 15 janvier 1970 : «On ne peut ignorer l'existence de quelques cas isolés de toxicomanie chez les élèves, cas entraînant, ou pouvant faire craindre, une contamination à l'intérieur de la collectivité scolaire. »

15. Pour une analyse des significations de l'usage du terme drogue au singulier, voir Beck, 2006.

16. Le «drame de Bandol » a fait entrer la toxicomanie dans l'agenda politique : la découverte d'une adolescente, morte par overdose dans les toilettes d'une station balnéaire varoise, suscite une couverture médiatique sans précédent et une indignation générale de l'opinion publique et de la classe politique (Bergeron, 1999). 
Dans la construction même d'un argumentaire interventionniste, tout se passe comme si la représentation médiatique du phénomène apparaissait suffisamment crédible pour rendre compte de son ampleur :

En marge des drames récents, dont nous avons accidentellement connaissance, on entrevoit la profondeur et l'accélération du mal. En fait, les affaires de drogue sont quatre fois plus nombreuses qu'en 1965 et concernent pour $30 \%$ des jeunes de moins de 21 ans et pour $9 \%$ des jeunes de moins de 18 ans : pour 100 drogués dans la population totale voici quatre ans, il y a aujourd'hui 120 drogués parmi les jeunes de moins de 21 ans, dont 36 de moins de 18 ans. Cette progression constitue pour la nation tout entière une menace très grave. (Ibid.)

Si la présentation des chiffres est confuse, un élément de quantification avéré s'en dégage : la part des usagers de drogues mineurs dénombrés par les services répressifs ne dépasse pas $10 \%$ de la population au début des années soixante-dix.

À l'occasion de ces débats parlementaires, certains députés pointent les limites des statistiques disponibles en soulignant les failles du système de repérage et de dénombrement par les services de police. Claude Marcus, député de l'Union des démocrates pour la République, évoque ainsi le fait que :

Les policiers arrêtent encore essentiellement des beatniks ou des hippies, facilement repérables, ou des jeunes dans des quartiers aisés à délimiter, comme SaintGermain-des-Prés, et voient donc un éventail assez restreint de drogués, de même les médecins ne traitent-ils que des intoxiqués parvenus à un stade avancé. Le développement de la consommation de certains toxiques chez des jeunes, parfois des très jeunes, dans des classes de lycée ou dans des lieux fort éloignés de Paris leur échappe encore presque totalement. Cela explique les importantes divergences statistiques [...]. Y a-t-il seulement de 3000 à 6000 drogués, comme le laissent entendre les services de police? Sont-ils au contraire plusieurs dizaines de milliers? La question demeure encore sans réponse, d'autant plus que la définition même du drogué reste à établir. (Compte rendu intégral de la séance de l'Assemblée nationale du 24 octobre 1969, Journal officiel du 25 octobre 1969, p. 2933)

Deux questionnements touchant à l'usage des chiffres sont soulevés dans cette intervention. Le premier porte sur la capacité des statistiques policières et sanitaires à rendre compte du phénomène d'usage de drogues dans son ensemble et dans sa diversité. Le second interroge la définition même de l'indicateur d'usage : qu'est-ce qu'un usage de drogue et qu'est-ce qu'un usager? Ces interrogations quant à ce que dénombrent les chiffres officiels se retrouvent dans l'intervention du député de l'Union centriste Jacques Barrot, qui rejoignait ce constat d'une mesure imprécise du phénomène :

En février 1968, M. le ministre de l'Intérieur [...] indiquait que les intoxiqués étaient au maximum un millier mais on relevait déjà parmi eux neuf étudiants et deux lycéens. [...] Le malheur veut que les statistiques exactes fassent défaut en 
la matière, que l'exploitation statistique des cas recensés n'ait pas encore été entreprise afin de mieux connaître, non seulement la dimension du phénomène, mais son impact sur les catégories socioprofessionnelles et sur les classes d'âge. (Compte rendu intégral de la séance de l'Assemblée nationale du 24 octobre 1969, Journal officiel du 25 octobre 1969, p. 2937)

En dépit de la méfiance exprimée à l'égard des chiffres, il concluait que si «il y a quelques années, il aurait été ridicule de parler d'un fléau social à propos de l'abus de stupéfiants ou de tout ce qu'on qualifie aujourd'hui de drogue, [...] nous sommes très près du fléau social en ce qui concerne les toxicomanies» (ibid.). À la fin des années soixante, la prise en compte par la classe politique du phénomène d'usage de drogues reposait donc sur des statistiques à la fois partielles, peu concluantes (deux lycéens et neuf étudiants sur l'ensemble du territoire) et structurellement fragiles.

Depuis le développement des enquêtes en population générale à partir de la fin des années quatre-vingt-dix, l'instrumentation des statistiques liées aux usages de drogues a redoublé les mécanismes d'inflation de l'ampleur du problème et renforcé le ciblage de l'action publique sur des populations particulières. L'ensemble des acteurs qui souhaitent communiquer sur les drogues disposent aujourd'hui d'un large choix d'indicateurs, qu'ils manient avec des "grilles de lecture » et des cadrages distincts, créant parfois des distorsions dans l'information offerte au grand public (Beck, 2005). Les chiffres, si fiables soient-ils, sont ainsi fréquemment enrôlés dans la mise en scène de situations parfois assez éloignées de la réalité qu’ils sont censés traduire.

\section{Le mythe de la précocité croissante de la consommation}

La précocité croissante de la consommation de drogues est devenue au cours des dernières années un thème récurrent des discours politiques, voire un "mythe », au sens de Roland Barthes, qui le définit tout à la fois comme un outil de réalisation des croyances et un système de communication, c'est-à-dire comme le signe d'une idéologie (Barthes, 1957, p. 216). Le rapport Drogues: l'autre cancer (Olin et Plasait, 2003), qui se donnait pour objectif d' "y voir plus clair dans la mise en œuvre de la politique nationale de lutte contre les drogues illicites, et surtout pour relever ses carences », parle ainsi d'un « rajeunissement général » des consommateurs, justifiant à ses yeux une politique plus volontariste de lutte contre l'usage :

Ce phénomène, sans doute le plus marquant de ces dernières années, a été souligné devant la commission par de nombreuses personnes auditionnées. Il recouvre plusieurs éléments : les jeunes connaissent leur primo-consommation de plus en plus tôt, de plus en plus de jeunes consomment et l'intensité des phénomènes de consommation chez les jeunes s'accroît avec l'âge. (Olin, Plasait, 2003, t. II, p.16 et 51) 
Ce postulat d'une précocité croissante de la consommation de drogues se retrouve dans le Plan gouvernemental adopté en 2008, qui affirme dans ses constats liminaires que « le rajeunissement de l'âge moyen d'initiation aux différentes substances psychoactives (11 ans pour l'alcool, 12 ans pour le tabac, 14-15 ans pour le cannabis) est l'une des tendances récentes les plus préoccupantes» (MILDT, 2008, p.15).

Alors que, selon les chiffres disponibles régulièrement actualisés, le rajeunissement du premier usage de cannabis semble limité aux toutes premières années de la décennie 2000 , le discours sur la précocité croissante des entrées en consommation constitue un invariant des discours publics depuis les années soixante. Ainsi, en 2011, la secrétaire d’État chargée de la jeunesse et de la vie associative, Jeannette Bougrab, affirmait-elle devant la mission commune sur les toxicomanies qu' " on observe un rajeunissement alarmant de l'âge moyen d'initiation aux différentes substances psychoactives » (sans préciser lesquelles), avant d'annoncer, sans crainte de l'amalgame, la commande d'un rapport au psychiatre Boris Cyrulnik sur le suicide des jeunes, arguant que «la consommation de substances psychoactives licites ou illicites constitue un appel au secours, un signal de détresse» (Branget, Barbier, 2011, t. II, p. 544). Loin d'être isolé, ce point de vue se retrouve dans les propos de plusieurs personnalités auditionnées par la mission, à l'instar du président de la Fédération nationale des associations de prévention de la toxicomanie qui, généralisant un constat limité à l'expérience au sein de son association, revendiquait l’idée d'une évolution massive du phénomène :

En 1996, les chefs d'établissement nous demandaient d'intervenir en seconde et en troisième ; en 1999, en quatrième et en cinquième, et en 2002, en CM2 ! Non seulement la consommation a explosé mais l'âge des premières consommations s'est abaissé à neuf ou dix ans. (Branget, Barbier, 2011, t. II, p. 104)

Outre le registre métaphorique employé par cet intervenant issu du champ de la prévention, par ailleurs président d'une association militante ${ }^{17}$, qui évoque l'image d'une «explosion » (rapportée dans les extraits d'audition avec un point d'exclamation) et des premières consommations dès l'âge de neuf ou dix ans (fait rarissime s'agissant des drogues illicites), on relève, dans les auditions diligentées par une instance parlementaire, l'immobilité des représentations. Récurrentes et affirmées quoique jamais documentées par des chiffres, celles-ci amènent la mission à conclure : « un rajeunissement des populations affectées serait néanmoins observé» (Branget, Barbier, 2011, t. I, p. 25).

17. L'association lilloise «Stop à la drogue », créée en 1996 «par des parents soucieux d'impliquer élèves, parents et enseignants dans un processus actif de prévention », se présente comme une structure non confessionnelle, composée de «180 familles adhérentes », visant à « développer l'esprit critique face à une culture de banalisation ». L'association est par ailleurs membre de l'Udaf (Union départementale des associations familiales) (sources : site de l'association et site www.famillechretienne.fr). 
Or, ni en 2003, ni en 2008, le « constat» d'un rajeunissement n'était clairement étayé par les statistiques, qui attestaient d'un phénomène déjà en cours de stabilisation (voir figure infra). En 2003, la baisse de l'âge moyen d'expérimentation du cannabis était peu marquée par rapport aux années précédentes; en 2008, cet âge moyen d'initiation avait déjà retrouvé son niveau de 2002. Sur une plus longue échéance (2000-2011), chiffres officiels à l'appui, l'initiation au cannabis n'est pas plus précoce qu'au début de la décennie. Pourtant, la mesure de cette évolution ne semble pas avoir été prise en compte dans les représentations du phénomène.

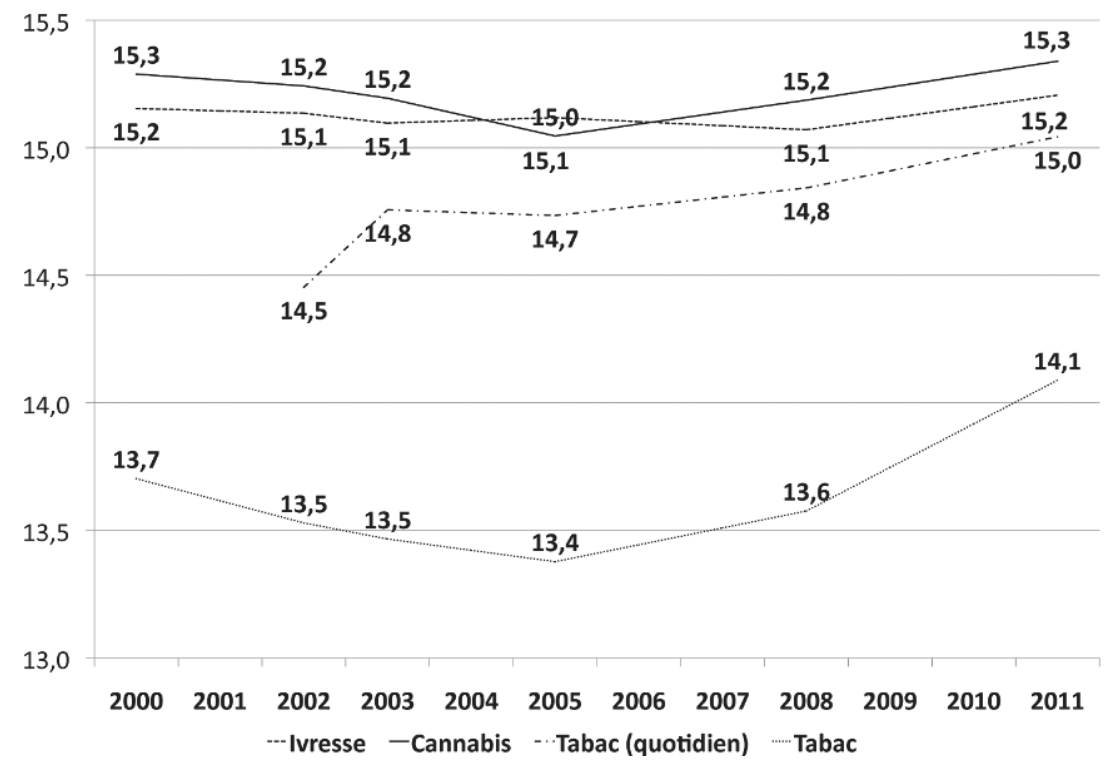

Source : Enquête ESCAPAD 2011, OFDT (Spilka et al., 2012).

Évolution de l'âge moyen d'expérimentation du tabagisme, de l'ivresse et de l'usage de cannabis (en années)

Cette focalisation sur les plus jeunes usagers de drogues et les indices de précocité de leurs expérimentations s'explique en partie par des préoccupations de santé publique orientées vers une catégorie prioritaire de la population : les jeunes, cible prioritaire des politiques publiques de lutte contre l'usage de drogues (Beck et al., 2010). La précocité des usages de substances psychoactives est en effet identifiée, tant par les études épidémiologiques que par les professionnels des addictions, comme un indice très défavorable dans la trajectoire d'un usager, souvent précurseur d'un usage problématique. 
Si une trop grande précocité est unanimement reconnue comme délétère, on peut néanmoins observer que l'indicateur mis en œuvre pour rendre compte de l'âge d'entrée en consommation (l'évolution de l'âge moyen) est davantage utilisé pour sa puissance rhétorique que pour étayer des faits avérés. La moyenne s'avère en effet être un outil assez médiocre pour analyser les évolutions de l'âge d'initiation, reflétant mal le phénomène à mesurer si la dispersion des valeurs est importante et les valeurs extrêmes nombreuses, ce qui est le cas dans les enquêtes en population générale adulte, par exemple. Dans ce cas, la moyenne se trouve affectée par les cas déviants et se distingue fortement de la médiane (qui, à l'inverse, incorpore des informations relatives à la valeur centrale de la distribution). Ainsi, pour étudier des pratiques sujettes à d'importantes variations individuelles, une analyse à partir de la distribution par âge pourrait être plus parlante. S'agissant de l'évolution de l'âge d'initiation aux drogues, pour peu que le nombre d'expérimentateurs s'élève dans la population générale, l'âge moyen d'entrée en consommation s'en trouvera probablement modifié à la hausse car la diffusion d'une pratique se traduit souvent par sa généralisation à des publics a priori moins intéressés par la pratique elle-même que les noyaux durs d'utilisateurs initiés les premiers. On peut par ailleurs percevoir, derrière le fantasme d'une précocité croissante des consommations de drogues qui ne saurait être attestée par des chiffres de long terme, le postulat rémanent selon lequel «la jeunesse n'est plus ce qu'elle était». Cette formule du sens commun a inspiré un article à Christian Baudelot (1988), qui analyse cette récrimination comme l'effet de la généralisation de droits aux jeunes. Pour Baudelot, la jeunesse a changé en ce que le statut social correspondant à la période de transition entre l'enfance et la vie adulte s'est modifié. Il souligne cependant que «la jeunesse» constitue un fait social multidimensionnel qui met à mal toute tentative de description, dans la mesure où elle ne peut se réduire au déroulement linéaire d'un calendrier unique : ainsi les processus d'insertion professionnelle s'enchevêtrentils, sans pourtant se confondre, avec les étapes de l'accession à l'autonomie résidentielle, financière, et celles de la vie affective et sexuelle. Pour historiciser les comportements de «la jeunesse» et décrire au plus près l'évolution des conduites d'expérimentation de drogues, il importe donc de «prendre de la hauteur» par rapport à l'idée, de courte vue, d'un rajeunissement de l'initiation aux substances psychoactives, en reconsidérant la question de la précocité au prisme des transformations qui ont affecté les conditions sociales du passage à l'âge adulte. Une telle approche suppose alors de prendre en compte l'ensemble des évolutions observées dans les comportements d'une classe d'âge, en les comparant aux comportements et aux conditions de vie des générations précédentes. 


\section{Le leitmotiv de la « dépénalisation de fait »}

Le rapport du Sénat paru en 2003 assimilait la politique de répression de l'usage menée à l'époque à une « dépénalisation de fait», jugeant «erratique» la réponse judiciaire donnée à ce contentieux de masse (Olin, Plasait, 2003, t. I, p.193). Parmi les propos repris pour soutenir ces conclusions, on relevait notamment ceux de Nicolas Sarkozy, ministre de l'Intérieur de l'époque, qui déclarait:

Tolérer la consommation est favoriser le travail des trafiquants, évidemment. Si le nombre de consommateurs augmente et si l'on peut tranquillement consommer, pourquoi voudriez-vous que les grands réseaux de trafiquants internationaux ne considèrent pas que notre pays est un lieu d'atterrissage, d'expansion et de commercialisation particulièrement sympathique, puisque vendre de la drogue est très mal, mais on ne dit rien si l'on en consomme? Si ce n'était si grave, nous pourrions parler d'incohérence. Depuis quelques années, le discours dénonce les trafiquants mais reste complaisant avec les usagers qui détiennent quelques grammes de cannabis ou quelques cachets, toujours pour leur consommation personnelle. C'est un illogisme absolu puisque, je l'affirme, il ne peut y avoir de trafic sans consommation. (Olin, Plasait, 2003, t. Il, p. 196)

Avançant le chiffre d'un taux de sanctions prononcées contre les usagers de drogues qui serait passé de «30\% en 1990 » à «à peine $8 \%$ en 2001 », il concluait que «pour 9 consommateurs sur 10, l'usage des stupéfiants s'est trouvé de fait dépénalisé», déplorant l'existence, dans la loi de 1970, de «sanctions disproportionnées pour un simple fumeur de cannabis et donc jamais appliquées» (Olin, Plasait, 2003, t. II, p. 543). La loi française prévoit en effet une peine maximale d'un an d'emprisonnement et de $3750 €$ d'amende pour usage simple de stupéfiants, sans distinction de produit.

Le leitmotiv d'une « complaisance » à l'égard des usagers visée dans ce discours se retrouve, plus récemment, dans les propos du président de la Fédération nationale des associations de prévention de la toxicomanie, auditionné en 2010-2011 par la Mission commune d'information sur les toxicomanies. Celuici dénonçait l'absence d'une réponse répressive systématique qui entretiendrait, selon lui, l'idée d'une relative impunité : «Les jeunes se droguent aussi parce qu'aucune sanction n'est jamais prononcée à l'encontre, ni des simples usagers, ni des petits “dealers”.» (Branget, Barbier, 2011, t. II, p. 105)

Confrontée aux chiffres officiels, cette représentation commune d'une répression «lacunaire et pusillanime » ${ }^{18}$ de l'usage de stupéfiants s'avère pourtant erronée. Si l'on rapproche les chiffres avant l'entrée dans la filière pénale (usagers interpellés) et à la sortie, en bout de chaîne (usagers condamnés), on obtient en

18. http://www.senat.fr/seances/s201112/s20111207/s20111207001.html, consulté le 20 septembre 2012. 
effet un ratio de un à sept, indice en apparence probant d'une application peu effective de la loi de 1970. Ainsi, en 2009 par exemple, près de 138000 personnes ont été interpellées pour usage et 20000 condamnations pour usage à titre principal 19 ont été prononcées (Timbart, 2009). Si l'on peut effectivement être tenté de rapporter le second chiffre au premier pour conclure à une faible pénalisation de l'usage, dès lors que $14 \%$ seulement des usagers interpellés sont condamnés, cette mise en relation chiffrée pose problème pour plusieurs raisons. Outre le fait qu'elle amène à comparer des chiffres établis sur des bases distinctes (Obradovic, 2012), la juxtaposition entre la délinquance commise (reflétée par le chiffre des interpellations) et celle sanctionnée par un jugement ignore une large partie des sanctions pénales prescrites par les parquets, qui ne constituent pas des condamnations prononcées par les tribunaux mais n'en demeurent pas moins des mesures pénales. Or, précisément, pour un contentieux de masse tel que l'usage de stupéfiants, considéré comme une "affaire simple ${ }^{20}$, le recours à ce maillon intermédiaire de la chaîne pénale joue un rôle central dans la gestion des flux. Ainsi, pour faire face à la hausse des interpellations pour usage simple, multipliées par six au cours des 25 dernières années (1986-2010), jusqu'à atteindre le chiffre record historique de près de 148000 procédures en 2008 , les acteurs de la justice pénale ont systématisé le recours aux mesures dites de la troisième voie (ou alternatives aux poursuites pénales). Rares jusqu'à la fin des années quatre-vingt-dix, ces mesures, dont la vocation est de traiter les infractions mineures autrefois classées sans suite, représentent aujourd'hui $70 \%$ des orientations prononcées par les procureurs pour sanctionner les usagers de stupéfiants (Obradovic, 2012). Elles peuvent prendre la forme d'un rappel à la loi, d'une injonction thérapeutique, d'une orientation sanitaire, etc.

Si la part des usagers interpellés faisant l'objet d'une condamnation judiciaire stricto sensu a en effet baissé au cours des deux dernières décennies, le niveau de pénalisation de l'usage de stupéfiants n'a donc pas pour autant reculé. Il atteint aujourd'hui un niveau inégalé du fait de la systématisation des sanctions alternatives au procès pénal pour les usagers interpellés, décidées par les parquets et non dans le cadre d'une condamnation. Le taux de réponse pénale aux infractions d'usage de stupéfiants dépasse ainsi $90 \%$.

Par ailleurs, même si le volume des condamnations pour usage a progressé moins rapidement que les interpellations au cours des deux dernières décennies, il a tout de même été multiplié par quatre. La part relative de l'usage parmi les condamnations pour infractions à la législation sur les stupéfiants a, de sur-

19. C'est-à-dire que l'infraction d'usage motive par elle-même la condamnation ou est considérée comme étant la plus grave d'un ensemble d'infractions.

20. Les procureurs de la République sont chargés d'orienter les procédures selon un principe de distinction entre les affaires «simples» (faisant l'objet de procédures rapides et simplifiées dans lesquelles le juge joue un rôle accessoire) et les procédures «traditionnelles» dans lesquelles le juge assure pleinement son rôle. Dans ce sens, les parquets constituent une plate-forme gestionnaire des politiques pénales (Milburn, 2010). 
croît, doublé entre 1990 et 2010 (passant de 18\% à 56 \%) et la part des condamnations pour usage sanctionnant cette seule infraction, hors de toute infraction associée, est passée de 62\% en 1990 à $76 \%$ en 2010 (Obradovic, 2012). Ainsi, dès lors que l'on définit la «pénalisation » comme l'ensemble des situations où l'usage de stupéfiants fait l'objet d'une sanction notifiée par la justice pénale ${ }^{21}$, on ne saurait valider l'assertion selon laquelle les usagers seraient rarement sanctionnés. On observe au contraire que l'usage de stupéfiants fait l'objet d'un traitement de plus en plus spécifique et ciblé, à l'image de la part croissante des condamnations pour usage sanctionnant cette seule infraction. L'idée reçue selon laquelle l'application de la loi serait peu effective, conduisant à une «dépénalisation de fait », constitue donc une bonne illustration de la dimension symbolique des discours sur le niveau de répression de l'usage de stupéfiants. Soutenir que la loi est peu appliquée répond à d'autres enjeux que ceux d'une "vérité scientifique », liés à des stratégies de positionnement politique. On peut en effet supposer qu'une telle affirmation vise à substituer à la "vérité des chiffres» un jugement qui relève de l'ordre normatif davantage que de l'ordre scientifique, qui insiste sur l'insuffisante pénalisation de l'usage par la loi (ou par son application). Dans ce sens, le discours sur la «dépénalisation de fait » opère un coup de force symbolique qui instaure un nouvel ordre du discours ${ }^{22}$, celui de la "tolérance zéro", où le fait que chaque infraction d'usage ne soit pas pénalisée équivaudrait à une situation où tous les usages de stupéfiants seraient permis. Ce qui est affirmé, à travers la dénonciation d'une « dépénalisation de fait», est bien l'idée qu'une sanction alternative aux poursuites ne constitue pas une sanction suffisamment sévère, selon les tenants d'une répression accrue de l'usage de stupéfiants. Cette position se trouve résumée dans le propos du ministre de l'Intérieur devant la commission d'enquête sénatoriale sur la politique nationale de lutte contre les drogues illicites en 2003. Appelant de ses vœux un discours «clair» de la part des pouvoirs publics sur la consommation de drogues, il déclarait souhaiter un «cadre législatif rénové », prévoyant des sanctions systématiques pour tous les types d'usages de drogues, sans distinction de produit ou de comportement d'usage ${ }^{23}$ :

21. Au sens d'un système de flux dont les unités de mesure sont les procès-verbaux, qui comptabilisent des types d'infractions (crime, délit, contravention) et les personnes mises en cause, qui feront l'objet d'une procédure parfois brève (classement sans suite, ordonnance pénale), parfois longue (instruction, procès criminel).

22. La référence à l'« ordre symbolique » du discours est empruntée à Gusfield, qui a analysé le processus de construction du problème public de «l'alcool au volant » aux États-Unis comme le produit des stratégies développées par divers acteurs (producteurs et distributeurs d'alcool, entreprises automobile, compagnies d'assurances) pour se défausser de toute responsabilité. La "production d'un ordre symbolique» a consisté à imposer « des croyances à propos de la factualité de la situation et des événements qui composent le problème », c'est-à-dire, en l'occurrence, la responsabilité du «conducteur-buveur» dans la survenue des accidents de la route (Gusfield, 1981, trad. fr. 2009).

23. Pour le ministre de l'Intérieur auditionné par la commission d'enquête, il n'y a ni drogue dure ni drogue douce : «Toutes sont nocives» (Olin, Plasait, 2003, t. II, p. 541). 
Je réclame la sévérité à l'égard des consommateurs. Rien ne sera toléré [...]. Il n’y a pas de drogue douce ou de drogue dure. Il n'y a pas de petite consommation personnelle, il n'y a pas d'expérience individuelle, il n'y a pas de jeunes «libres et branchés ", il n'y a que des drogues interdites et des usagers qui [...] transgressent la loi. (Olin, Plasait, 2003, t. II, p. 541)

Afin d'instaurer un «dispositif réellement applicable », le ministre revendiquait la «création d'une échelle de sanctions adaptées qui permettent de punir réellement et rapidement tant les mineurs qui consomment occasionnellement du cannabis ou de l'ecstasy que les usagers d'héroïne qui refusent les soins ». Tout se passe comme si la mobilisation du chiffre des usagers condamnés (plutôt que des usagers sanctionnés en amont des tribunaux correctionnels) permettait d'euphémiser la teneur idéologique d'un propos en masquant son caractère intrinsèquement normatif, les statistiques étant réputées «gage d'impartialité »24. Ces stratégies d'enrôlement du chiffre, qui visent ici à légitimer un surcroît d'efforts répressifs ${ }^{25}$, trouvent évidemment leur pendant « antisécuritaire » dans des usages concurrents de statistiques qui tendent à mettre en avant d'autres manières d'appréhender le problème ou une autre lecture des mêmes sources.

Il faut souligner enfin que, si le recours aux chiffres contribue à produire et à structurer des cadres d'analyse du «problème de la consommation de drogues », ce cadrage est alimenté en retour par les représentations sociales. Les usages rhétoriques du chiffre par les acteurs politiques sont en effet irrigués par les représentations de l'opinion publique sur les drogues et ceux qui les consomment ${ }^{26}$, qui agissent comme des contraintes (ou des incitations) à développer des stratégies politiques identifiables.

L'évaluation des politiques menées pour lutter contre l'usage de drogues apparaît comme un enjeu crucial de communication politique : elle participe d'une volonté de faire adhérer l'opinion publique aux choix politiques soumis à l'épreuve de l'évaluation chiffrée (Gerstlé, 2008, p. 79). Elle met en évidence des entreprises de qualification (linguistique, juridique, normative) de la nature et des termes du problème posé par la consommation de drogues chez les jeunes. Le recours à certains chiffres (plutôt que d'autres) joue un rôle central dans ces luttes symboliques, qui reposent en partie sur la capacité à imposer des arguments faisant autorité. Or la valorisation d'un chiffre contri-

24. Voir, sur ce point, les travaux de Theodore Porter (1995) sur la « confiance dans les nombres».

25. Notons, pour appuyer cette hypothèse sur l'usage stratégique des chiffres, que Nicolas Sarkozy, interrogé par la commission parlementaire en 2003 sur le traitement pénal à réserver aux usagers de stupéfiants, soulignait de sa propre initiative : "Ce sont des chiffres. Après, chacun les exploitera, leur fera dire ce qu'il veut, mais j’observe. » (Olin, Plasait, 2003, t. II, p. 543)

26. Les représentations sociales sur les drogues sont par exemple mesurées par l'enquête EROPP (Enquête sur les représentations, opinions et perceptions sur les psychotropes) menée par l'OFDT. 
bue à construire le problème en même temps qu'il impose la nécessité pour les pouvoirs publics de s'en saisir. L'analyse de la portée symbolique des chiffres mobilisés dans le débat sur les jeunes face aux drogues qui est proposée dans cet article illustre comment l'usage sélectif des chiffres constitue un instrument de transformation politique et sociale autant qu'un outil de mesure objectif.

\section{Références}

BARThes Roland, 1957, Mythologies, Paris, Le Seuil.

BAUdelot Christian, 1988, «La jeunesse n'est plus ce qu'elle était. Les difficultés d'une description », Revue économique, vol.XXXIX, nº 1, janvier, p.189-225.

BECK François, 2005, "Dénombrer les usagers de drogues. Tensions et tentations», Genèses, nº 1, vol. LVIII, p. 72-97.

— 2006, «Entre représentativité des échantillons et représentation des usages. L'apport des enquêtes en population générale à la compréhension des usages de drogues », thèse de doctorat de sociologie, Université René Descartes Paris V, 478 p.

Beck François, Obradovic Ivana, Jauffret-Roustide Marie, Legleye Stéphane, 2010, «Regards sur les addictions des jeunes en France», Sociologie, vol. I, nº 4, p. 517-536.

BECKER Howard S. éd., 2001, Qu'est-ce qu'une drogue?, Atlantica, Anglet.

Bergeron Henri, 1999, L'État et la toxicomanie. Histoire d'une singularité française, Paris, PUF.

Bernat de CÉlIS Jacqueline, 1994, Consommation interdite. Un délit d'usage des stupéfiants, pourquoi ?, Paris, La Documentation française.

BouRdieu Pierre, 1984, Questions de sociologie, Paris, Minuit.

BRANGET Françoise, BARBIER Gilbert, 2011, Rapport d'information no 699 (2010-2011) sur les toxicomanies, fait au nom de la Mission d'information sur les toxicomanies, déposé le 30 juin 2011, Sénat-Assemblée nationale, http://www.senat.fr/rap/r1o699-1/r10-699-11.pdf.

CHAMBoredon Jean-Claude, 1966, «La société française et sa jeunesse », Le partage des bénéfices, E. Darras éd., Paris, Minuit.

Degenhardt Louisa, Conroy Elizabeth, Gilmour Stuart, Collins Linette, 2005, «The effect of a reduction in heroin supply in Australia upon drug distribution and acquisitive crime », British Journal of Criminology, vol. XLV, p. 2-24.

Desrosı̇̀res Alain, 2004, "Pour une politique des outils du savoir. Le cas de la statistique», communication à la Conférence «Politics and Knowledge. Democratizing knowledge in times of the expert », Université de Bergen, 21 au 24 juin 2004.

- 2008, Pour une sociologie historique de la quantification, Paris, Presses de l’École des mines.

GeRSTLÉ Jacques, 2008 [2004], La communication politique, Paris, Armand Colin.

Gusfield Joseph, 1981, The Culture of Public Problems. Drinking-Driving and the Symbolic Order, Chicago, The University of Chicago Press (La culture des problèmes publics. L'alcool au volant : la production d'un ordre symbolique, trad. D. Cefaï, Paris, Economica, 2009). 
Houllzon Philippe, 2010, "Le contrôle extraordinaire du Parlement», Pouvoirs, 2010/3, no 134, p. 59-69.

Kopp Pierre, Fenog lio Philippe, 2004, Coûts et bénéfices économiques des drogues en France, Paris, OFDT.

LAHAYE Didier, 1989, Drogue, toxicomane, toxicomanie. La production des catégorisations et leurs enjeux sociaux, Paris, Mémoire de DEA en sciences sociales, ENS, EHESS.

Layne Mary, Bruen Ann-Marie, Johnson Patrick, Rhodes William, DeCKer Scot, TownSEnd Meg, Chester Caben, Schaffer Gary, LAVIn John, 2001, Measuring the Deterrent Effect of Enforcement Operations on Drug Smuggling, 1991-1999, Washington, Abt Associates.

MILbuRn Philip, 2010, «Les procureurs de la République. Passeurs de justice ou gestionnaires des politiques pénales? », Droit et société, $\mathrm{n}^{\circ}$ 74, p. 73-90.

MiLdT (Mission interministérielle de lutte contre la drogue et la toxicomanie), 1999, Plan triennal de lutte contre la drogue et de prévention des dépendances 19992001, Paris, La Documentation française, MILDT.

- 2004, Plan gouvernemental de lutte contre les drogues illicites, le tabac et l'alcool 2004-2008, Paris, La Documentation française, MILDT.

- 2008, Plan gouvernemental de lutte contre les drogues et les toxicomanies (20082011), Paris, La Documentation française, MILDT.

OBRADOVIC Ivana, 2012, «La pénalisation de l'usage de stupéfiants en France au miroir des statistiques administratives. Enjeux et controverses», Déviance et société, vol.XXXVI, no ${ }_{4}$, vol.XXXVI, p. 437-465.

Olin Nelly, Plasait Bernard, 2003, Drogue : l'autre cancer. Rapport d'information 321 sur la question des drogues, t. I (2002-2003), Paris, Sénat.

PORTER Theodore, 1995, Trust in Numbers. The Pursuit of Objectivity, in Science and Public Life, Princeton, Princeton University Press.

Reuter Peter, GReen field Victoria A., 2001, «Measuring global drug market. How good are the numbers and why we should care about them?", World Economics, vol.II, no 4 , p. 159-173.

Setbon Michel, GuÉrin Olivier, KARSEnty Serge, Kopp Pierre éd., 2003, Évaluation du plan triennal de lutte contre la drogue et de prévention des dépendances (19992002). Rapport général, Paris, OFDT.

SPILKA Stanislas, Le NÉzet Olivier, Tovar Marie-Line, 2012, « Les drogues à 17 ans. Premiers résultats de l'enquête ESCAPAD 2011 », Tendances, n079, OFDT.

THÉVENOT Laurent, 1979, "Une jeunesse difficile. Les fonctions sociales du flou et de la rigueur dans les classements", Actes de la recherche en sciences sociales, n० $26 / 27$, mars-avril.

TIMBART Odile, 2009, Les condamnations. Provisoires, Année 2008, Paris, Ministère de la Justice, Sous-direction de la statistique.

Wood Evan, Tyndall Mark W., SPItTAl Patricia, Lı Kathy, Anis Aslam H., Hog Robert S., Montaner Julio S. G., O’Shaughnessy Michael V., SCheChter Martin T., 2003, "Impact of supply-side policies for control of illicit drugs in the face of AIDS and overdose epidemics. Investigation of a massive heroin seizure », Canadian Medical Association Journal, vol. CLXVIII, nº 2, p.165-169. 